\title{
ASTEROID MASS DISTRIBUTION MEASUREMENT WITH GRAVITY GRADIOMETERS
}

\author{
ROBERT L. FORWARD \\ Hughes Research Laboratories
}

A determination of the internal structure and composition of the asteroids will give us important information concerning the origin of the solar system and the formation of the planets. We can obtain this information by measuring the total mass and the internal mass distribution through the use of spacecraft missions to flyby or to rendezvous, orbit, and land on the asteroids. The Doppler tracking technique used for mass measurement on planetary probes is suitable only for a flyby mission of the larger asteroids $(>20 \mathrm{~km})$ because the gravity force field of the smaller asteroids is not strong enough to appreciably affect the trajectory of the probe during a flyby. If a rendezvous mission is used and the spacecraft is placed in orbit about an asteroid, the mass can be determined from the orbital period, but the effect on the spacecraft orbit due to the mass anomalies under the surface will not be easily seen unless the anomaly is very large because again the gravity force field decreases rapidly with decrease in anomaly size. The magnitude of the gradient of the gravity force field is independent of the asteroid or anomaly size, however, and a gravity gradient sensing technique for sensing of the gravity field of the asteroid will perform equally well on all except the very smallest $(<1 \mathrm{~km})$ asteroids. Thus, if we desire to obtain mass measurements of the smaller asteroids during a flyby or to obtain detailed mass anomaly maps of the asteroids from an orbital survey prior to landing, it would be desirable to include a gravity gradiometer as part of the spacecraft instrument package.

\section{GRAVITY GRADIOMETER INSTRUMENTATION}

The various gravity gradient instruments that could be used to measure the gravity gradient field of an asteroid from a spacecraft all work on the principle of measuring the differential tensions, compressions, or torques induced in the sensor by the gradient of the gravity force field (the tidal effect) of the asteroid. There are many techniques and configurations possible (a fairly complete bibliography is available in (Bell, Forward, and Williams, 1970), but the instruments presently under serious consideration for spacecraft missions are those that measure the gravity gradient torque. These torque gradiometers 
use the same physical principle for their operation as the gravity gradient stabilized satellites. The gravity gradient satellites we are familiar with have an elongated dumbbell shape and are very large and massive in an attempt to obtain engineeringly significant torque levels out of the gravity gradient field. The gradiometers are smaller so that they can be enclosed for protection from nongravitational forces, and therefore must have high sensitivity. The typical signal levels encountered by these instruments range from $10^{-9}$ to $3 \times 10^{-6} \mathrm{~s}^{-2}$ ( $(1$ to 3000 Eötvös units (EU)) and the present designs have a noise level of $10^{-9} \mathrm{~s}^{-2}$ at $10 \mathrm{~s}$ integration time (Bell, Forward, and Williams, 1970).

One type of torque gradiometer is a single dumbbell in a cylindrical or spherical case that is floated at neutral buoyancy using the techniques developed for high-precision floated gyros (Trageser, 1970). Highly sensitive torque pickoffs and electromagnetic servodrivers are used to keep the float balanced and to read out the gravity gradient torques. (See fig. 1.)

A second technique, based on free-fall modifications of the old Eötvös torsion balance gradiometer, would use one or more dumbbells connected by fine quartz torsion fibers with capacitive pickoffs and electrostatic feedback. The gravity gradient signal would be that of the relative torque between the two sensing arms. (See fig. 2.)

A third variation also uses two opposed dumbbells, but they are connected by a stiff torsion spring that forms a resonant mechanical structure. (See fig. 3.) The entire device is then rotated at 60 to $1800 \mathrm{rpm}$ so that the gravity gradient field is "chopped" by the rotating arms (Bell, Forward, Williams, 1970; Forward, Pilcher, and Norwood, 1967). The resonant frequency of the sensor structure is chosen at twice the rotation frequency, and the gravity gradient field induces vibrations into the rotating sensor structure at its

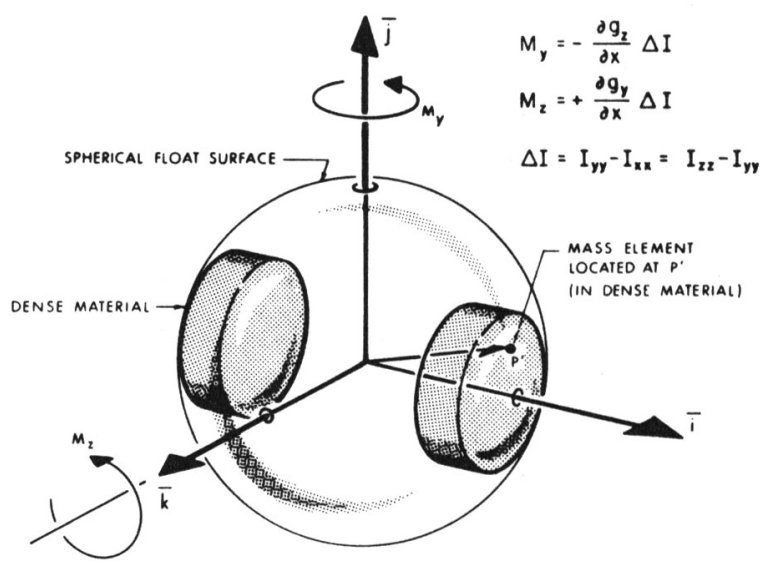

Figure 1.-Schematic of spherical floated torque gradiometer (from MIT Charles Stark Draper Laboratory, Cambridge, Mass.). 


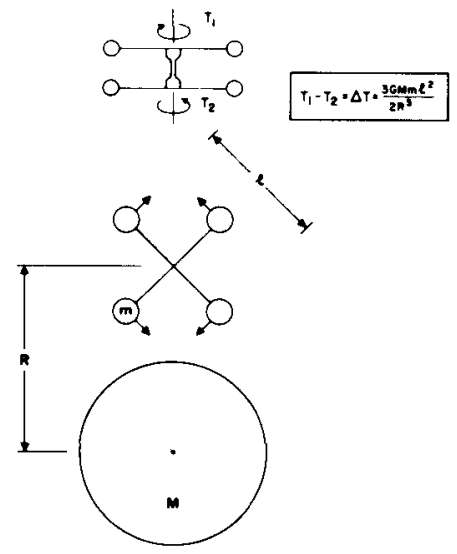

Figure 2.-Differential gravity gradient torques on a two-dumbbell torque gradiometer sensor structure. (The rotation axis of the rotating version of this type of gradiometer structure would be out of the page.)

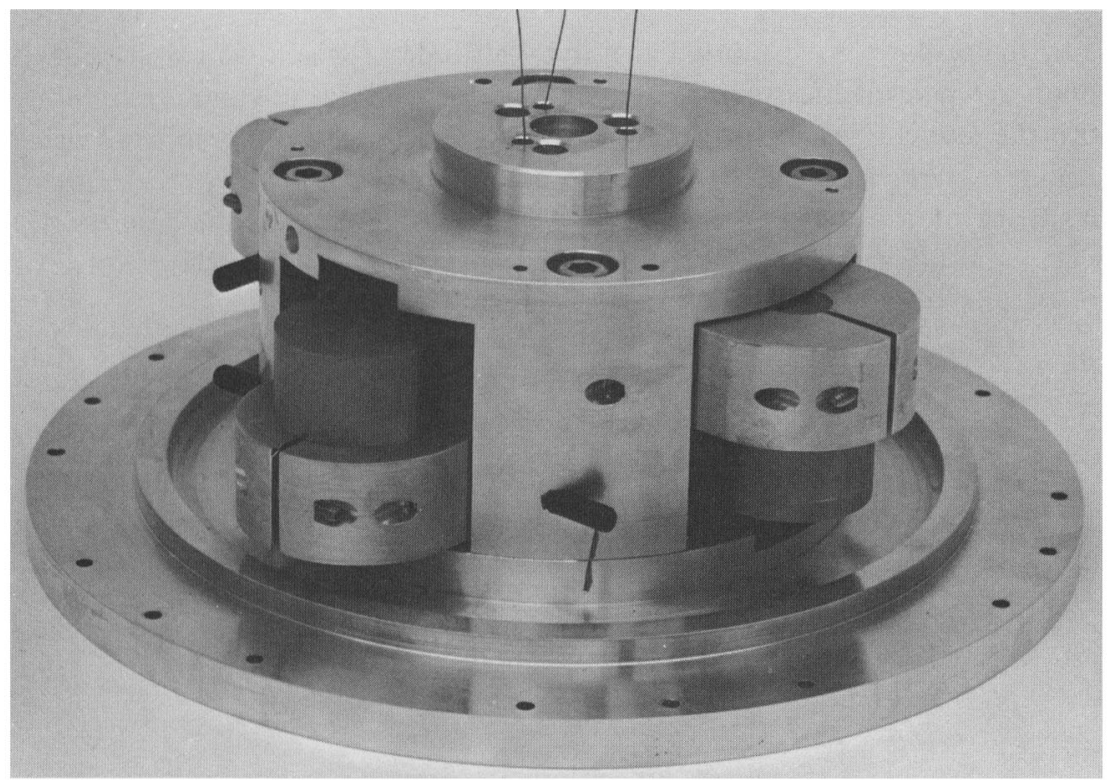

Figure 3.- Rotating gravity gradiometer.

resonant frequency. Piezoelectric transducers on the torsion spring convert the resonant mechanical vibrations into ac voltages whose amplitude and phase give the strength and direction of the gravity gradient field. If the rotating gradiometer is installed in a spin-stabilized spacecraft and the sensor resonance 
is tuned to twice the spacecraft rotation frequency, the spacecraft then provides the rotation needed for the sensor operation. This mode of operation has the advantage that because the spacecraft is rotating along with the sensor, its gravity field is fixed with respect to the sensor arms and the sensor does not see the spacecraft gravity field, only the asteroid field.

The various possible versions of the gravity gradiometer are just leaving the laboratory and are far from being tested flight hardware, but we can expect that by the time the asteroid missions begin, the instrumentation will be available. However, the gradiometer instrumentation adds significantly to the cost, weight, and power budgets of the spacecraft, whereas Doppler tracking is practically free. Therefore, the gravity gradiometer instrumentation should only be included on those missions for which the Doppler tracking data are not adequate for determination of the mass or mass distribution. In the following sections we will try to give some general guidelines that show when one technique is preferred over the other. This hopefully will help those who are planning the missions to obtain the maximum scientific return from each flight.

\section{MASS MEASUREMENT DURING A FLYBY}

In figure 4 we have plotted the flyby altitude at which we can expect to obtain a 1 percent measurement of the mass of asteroids of various radii using both the Doppler velocity tracking technique and the gravity gradient sensing technique. We assumed that the accuracy limit was set by the present sensitivity of the two systems, $10^{-9} \mathrm{~s}^{-2}(1 \mathrm{EU})$ at $10 \mathrm{~s}$ for the gradiometer system and $0.5 \mathrm{~mm} / \mathrm{s}$ at $60 \mathrm{~s}$ for the Doppler tracking system. The purpose of

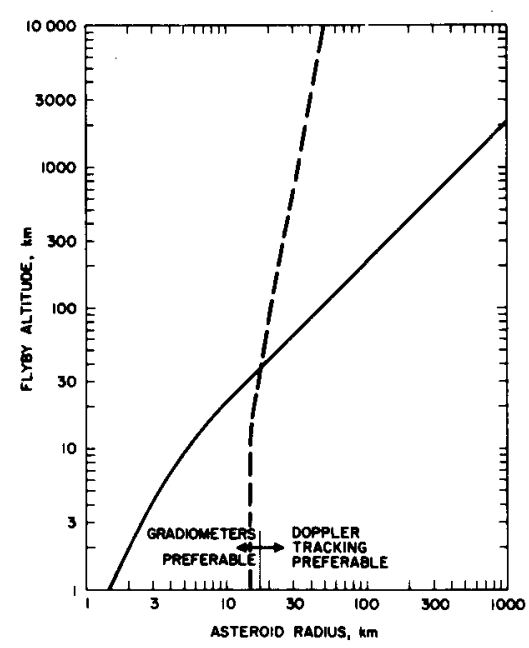

Figure 4.-Flyby altitude for 1 percent mass measurement (flyby velocity $=1 \mathrm{~km} / \mathrm{s}$ ) using Doppler tracking (broken curve) and gravity gradiometer techniques (solid curve). 
this graph is not to say that one technique is better than another, but to bring out the general features of the advantages and disadvantages of the two complementary techniques. This graph is for a flyby velocity of $1 \mathrm{~km} / \mathrm{s}$. Other curves with flyby velocities from 0.1 to $10 \mathrm{~km} / \mathrm{s}$ show the same general result. As we can see, the Doppler tracking technique is preferable for the larger asteroids, but becomes quite insensitive for the smaller asteroids, even for very close flybys. This general conclusion that the Doppler tracking technique is not suitable for flyby missions to the smaller asteroids is discussed in greater detail in the paper by John D. Anderson in these proceedings. ${ }^{1}$ (The Doppler tracking curve in fig. 4 is taken from fig. 3 in his paper.) The gravity gradiometer technique will give results for all asteroids above $1 \mathrm{~km}$, but its measurement range for the larger asteroids is poorer than the Doppler tracking technique. Future increases in the accuracy of either system will not change these general conclusions significantly because of the rapid falloff of both curves. A change in sensitivity of an order of magnitude will only shift the curves a factor of 2 in asteroid radius or in flyby altitude.

\section{ASTEROID RENDEZVOUS MISSIONS}

When we investigate techniques for mass. measurement and mass anomaly measurement that are applicable to a rendezvous mission to an asteroid, we find four techniques that can be considered: orbital velocity tracking, orbital period measurement, gravity gradient measurement, and acceleration measurement (after landing). All of these techniques can give accurate measurement of the mass of the larger asteroids, although the orbital velocity and the accelerometer techniques become less accurate for the smaller asteroids.

The orbital velocity of a spacecraft about an asteroid

$$
v=(G M / R)^{1 / 2}
$$

ranges from $300 \mathrm{~m} / \mathrm{s}$ for Ceres to $9 \mathrm{~mm} / \mathrm{s}$ for a $10 \mathrm{~m}$ radius asteroid. The velocity about the larger asteroids is high enough that present Doppler velocity tracking techniques are more than adequate for an accurate mass measurement. For the smaller asteroids, however, it is better to measure the time for one orbital period rather than the orbital velocity directly. The orbital period for a close orbit is independent of the mass of the asteroid and is a function of the average asteroid density

$$
T=(3 \pi / G \rho)^{1 / 2}
$$

The period ranges from about $3 \mathrm{hr}$ for an iceball to $1 \mathrm{hr}$ for a very dense asteroid.

${ }^{1}$ See p. 577. 
If the rendezvous vehicle lands on the asteroid, then we can use gravimeters or the spacecraft navigation accelerometers to measure the gravity force to obtain an estimate of the mass. The acceleration field

$$
a=G M / R^{2}
$$

ranges from $0.03 \mathrm{~g}$ for Ceres to $10^{-6} \mathrm{~g}$ for a $10 \mathrm{~m}$ radius asteroid. Although quite small, these acceleration levels can be measured to high accuracy by any number of available accelerometers and gravimeters. Both the accelerometer technique and the orbital period technique, however, are limited to obtaining an estimate of the total mass or average density of the asteroid. If we are interested in obtaining data on the internal density distribution of the asteroid, the use of the Doppler velocity tracking and the gravity gradiometer techniques from orbit are most suitable.

The horizontal gravity gradient of an asteroid is

$$
\Gamma=\frac{G M}{R^{3}}=\frac{4}{3} \pi G \rho
$$

If the gradiometer is in a close orbit about the asteroid so that the distance from the center of the asteroid is nearly equal to the radius, then the gravity gradient is only a function of the average asteroid density, and varies from $6 \times 10^{-7} \mathrm{~s}^{-2}(600 \mathrm{EU})$ for an iceball to $5 \times 10^{-6} \mathrm{~s}^{-2}(5000 \mathrm{EU})$ for a very dense asteroid.

The data that can be obtained on the internal mass distribution of an asteroid from Doppler tracking and gravity gradiometer measurements using an orbiting vehicle are compared in the following figures. Figure 5 shows a schematic of the hypothetical asteroid that was used in the computer simulations. The asteroid is $100 \mathrm{~km}$ in radius and has an average density of 3.5 $\mathrm{g} / \mathrm{cm}^{3}$. Embedded in this asteroid are spherical mass anomaly regions with radii of $1,3,10$, and $30 \mathrm{~km}$ and a density difference of $0.5 \mathrm{~g} / \mathrm{cm}^{3}$. If the orbiting

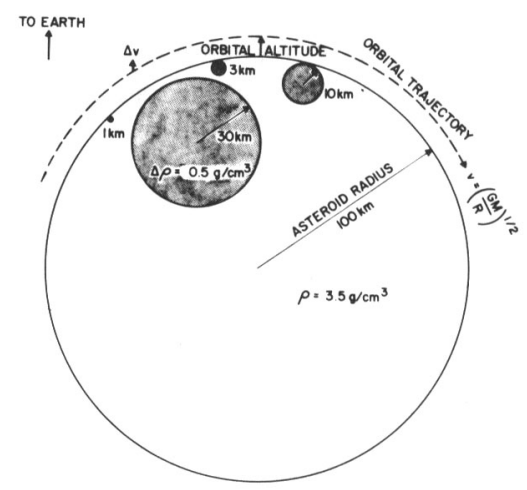

Figure 5.-Asteroid model used in computer simulations. 
vehicle is $1 \mathrm{~km}$ above the surface, then the output of the Doppler tracking system and the gravity gradiometer system during the passage over the anomalies is as shown in figure 6. At this altitude we can see that the gradiometer system gives significantly improved resolution and signal level for the smaller anomalies. If the altitude is raised to $3 \mathrm{~km}$ (to possibly avoid collision with the surface features), then we obtain the comparative plots shown in figure 7 . The advantage of the gradiometer data is now not so

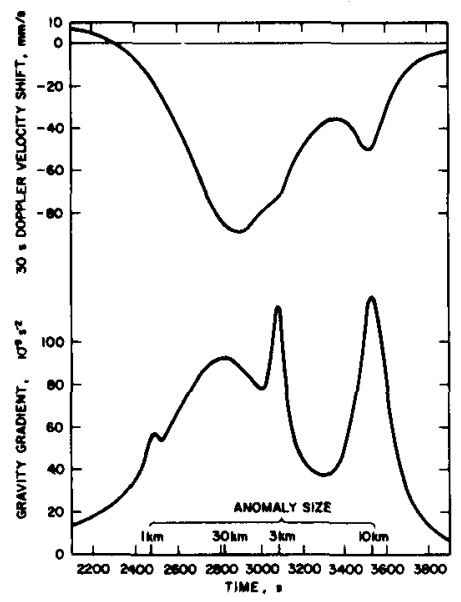

Figure 6.-Gravity gradient and Doppler tracking signal variations from an orbit $1 \mathrm{~km}$ above a $100 \mathrm{~km}$ radius asteroid.

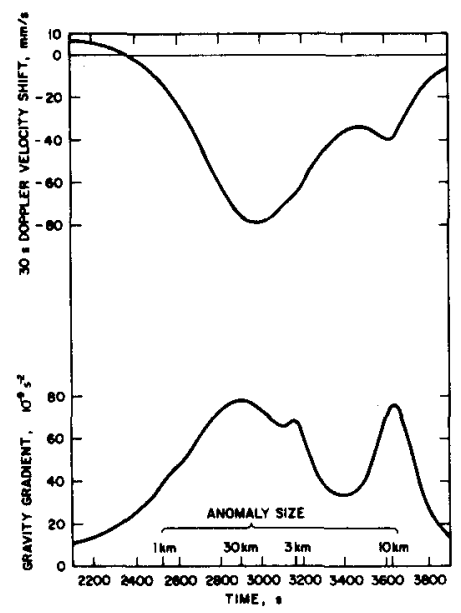

Figure 7.-Gravity gradient and Doppler tracking signal variations from an orbit $3 \mathrm{~km}$ above a $100 \mathrm{~km}$ radius asteroid. 


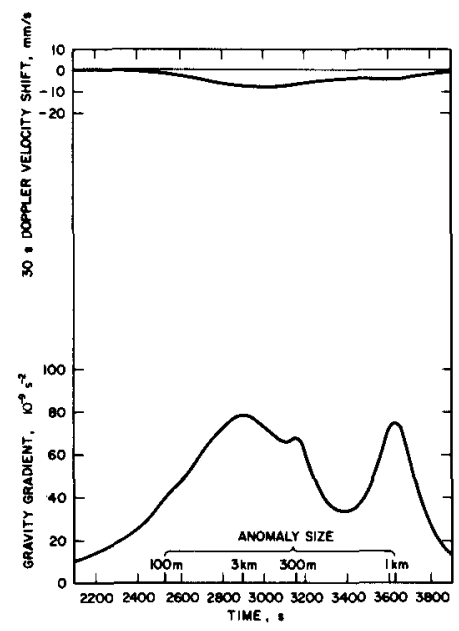

Figure 8.-Gravity gradient and Doppler tracking signal variations from an orbit $300 \mathrm{~m}$ above a $10 \mathrm{~km}$ radius asteroid.

significant, and the advantages of the slight improvement in data must be weighed against the costs. The significant advantage of the gradiometer technique is shown in figure 8 where we have assumed a decrease in scale of the simulation by a factor of 10 . Instead of a spacecraft in an orbit $3 \mathrm{~km}$ above a $100 \mathrm{~km}$ asteroid with 1 to $30 \mathrm{~km}$ sized anomalies, we have simulated a spacecraft in an orbit $300 \mathrm{~m}$ above a $10 \mathrm{~km}$ asteroid with 100 to $3000 \mathrm{~m}$ sized anomalies. The orbital period has not changed, because the asteroid density is assumed to be the same, so the time required for the measurement is the same. The gravity gradient signal has the same magnitude and resolution for the 10 $\mathrm{km}$ asteroid as it had for the $100 \mathrm{~km}$ asteroid, but the Doppler velocity signal has decreased by an order of magnitude and the accuracy of this technique for mass anomaly measurement has decreased in the same proportion.

\section{SUMMARY}

As a general rule, our studies show that the average density of an asteroid can best be obtained by Doppler tracking techniques if the mission is a flyby mission to one of the larger asteroids. If the mission involves a flyby of a smaller asteroid, or a rendezvous and orbit of any asteroid, the addition of a gravity gradiometer to the spacecraft instrument package will give a significant improvement in the quality of the gravity data and should be seriously considered for such missions.

\section{REFERENCES}

Bell, C. C., Forward, R. L., and Williams, H. P. 1970, Simulated Terrain Mapping With the Rotating Gravity Gradiometer. Proc. Invitational Symp. Dyn. Gravimetry (Fort Worth), Mar. 16-17, pp. 45-60. 
Forward, R. L., Pilcher, L. S., and Norwood, Virginia T. 1967, Asteroid Belt Investigation Using Small, Spin-Stabilized Fly-By Probes. Proc. AAS Symp. Planet. Geol. Geophys. (Boston), May 25-27, pp. 327-347.

Trageser, M. B. 1970, A Gradiometer System for Gravity Anomaly Surveying. Proc. Invitational Symp. Dyn. Gravimetry (Fort Worth), Mar. 16-17, pp. 1-43.

\section{DISCUSSION}

HARRIS: With a gravity gradiometer is there an ambiguity in the density distribution? FORWARD: There is always a mathematical ambiguity in the details of the internal density distribution of an object obtained from external gravity data alone. The ambiguity will have to be resolved with additional data obtained from magnetic, acoustic, or borehole surveys along with reasonable assumptions for the types of materials (rock, iron, ice, etc.) 